\title{
Molecular allelokaryotyping of relapsed pediatric acute lymphoblastic leukemia
}

\author{
NORIHIKO KAWAMATA ${ }^{1 *}$, SEISHI OGAWA ${ }^{2 *}$, KARL SEEGER $^{3}$, RENATE KIRSCHNER-SCHWABE $^{3}$, \\ THIEN HUYNH ${ }^{1}$, JOHN CHEN ${ }^{1}$, NAIRI MEGRABIAN ${ }^{1}$, JOCHEN HARBOTT $^{4}$, MARTIN ZIMMERMANN $^{5}$, \\ GÜNTER HENZE ${ }^{3}$, MARTIN SCHRAPPE $^{6}$, CLAUS R. BARTRAM ${ }^{7 * *}$ and H. PHILLIP KOEFFLER ${ }^{1 * *}$ \\ ${ }^{1}$ Hematology/Oncology, Cedars-Sinai Medical Center/UCLA School of Medicine, Los Angeles, CA, USA; \\ ${ }^{2}$ Regeneration Medicine of Hematopoiesis, University of Tokyo, School of Medicine, Tokyo, Japan; \\ ${ }^{3}$ Department of Pediatrics - Oncology/Hematolgy, Charité - Universitätsmedizin Berlin, Berlin; ${ }^{4}$ Department of Pediatric \\ Hematological Oncolcogy, Justus Liebig University, Giessen; ${ }^{5}$ Department of Pediatric Hematology and Oncology, \\ Children's Hospital, Hannover Medical School (MHH), Hannover; ${ }^{6}$ Department of Pediatrics, University of Kiel, Kiel; \\ ${ }^{7}$ Institute of Human Genetics, University of Heidelberg, Heidelberg, Germany
}

Received December 4, 2008; Accepted January 26, 2009

DOI: 10.3892/ijo_00000290

\begin{abstract}
Acute lymphoblastic leukemia (ALL) cells at relapse are frequently more resistant to treatment than primary clones and this may be caused by further genetic changes in the ALL cells at relapse. These acquired genomic abnormalities have not been fully characterized. To examine the additional genomic alterations of ALL at relapse, we performed single nucleotide polymorphism genomic microarry (SNP-chip) analysis on 14 ALL bone marrow samples at initial diagnosis, remission and relapse. Only two cases at initial diagnosis had a normal appearing genome by SNP-chip. All 14 cases had genomic alterations at relapse; and 10 of these had additional genomic abnormalities not present at diagnosis. Deletion of either the INK4A/ARF gene (2 cases) or the NF2 gene (2 cases) at 22q12.2 was an acquired genomic change at relapse. Loss of heterozygosity with normal copy number [uniparental disomy (UPD)] was detected in 3 cases as an additional genomic change at relapse. Interestingly, several genomic alterations, especially deletions, detected at initial diagnosis, disappeared at relapse, suggesting the ALL cells at relapse were minor clones at initial diagnosis and emerged at relapse. For several cases, trisomy at initial diagnosis changed to either UPD (2 cases) or normal appearing genome ( 2 cases). Further, we found disruption of PTPRD gene occurring at
\end{abstract}

Correspondence to: Dr Norihiko Kawamata, Hematology/ Oncology, Cedars-Sinai Medical Center/UCLA School of Medicine, 8700 Beverly Blvd., Los Angeles, CA 90048, USA

E-mail: nkawamata@gmail.com

${ }^{*}$ Contributed equally as the first authors

${ }^{* *}$ Contributed equally as the last authors

Key words: single nucleotide polymorphism genomic microarry, uniparental disomy, INK4A/ARF, NF2, PTPRD intron 23 as an additional genomic abnormality in one case. In summary, additional genomic changes are very common events in ALL at relapse; whether these abnormalities are associated with resistance to treatment remains to clarified in further studies.

\section{Introduction}

Pediatric acute lymphoblastic leukemia (ALL) is the most common malignant disease of children $(1,2)$. Improvement of treatment for this disease has achieved disease-free survival of $80 \%$ or more at 5 years (2-4). However, one fifth of patients relapse and are often refractory to chemotherapeutic reagents (2-4). Several clinical and genetic factors have been reported to be associated with refractoriness to treatment including high white blood cell count at diagnosis, BCRABL abnormality, hypodiploidy and short first complete remission $(1,2)$.

Studies have suggested that chemo-sensitivity of ALL cells in vitro at initial diagnosis is a good indicator for long-term survival $(5,6)$. Also, gene expression profiling of ALL cells is associated with their genetic status and chemo-sensitivity in vitro $(7,8)$. Genetic abnormalities of ALL cells influence their gene expression pattern and may be associated with their chemo-sensitivity (9).

After relapse, the leukemic cells are often refractory to standard chemotherapeutic reagents and more aggressive therapies are needed to treat this resistant disease, including hematopoietic stem cell transplantation $(10,11)$. This refractoriness may be attributed to acquired additional genetic abnormalities in the leukemic cells during relapse. Genomic differences between initial and relapsed ALL have not been fully examined.

Recently, single nucleotide polymorphism genomic microarray (SNP-chip) has been developed $(12,13)$. This new technique allows us to analyze genomic status of leukemic cells at very high resolution (molecular level) and allelespecific gene dosage levels (paternal and maternal alleles) can 
be examined (14-16). We named this technique molecular allelokaryotyping $(15,16)$. We used it on matched samples obtained at diagnosis, bone marrow remission and relapse in order to identify genomic differences between initial and relapsed ALL and to find genes associated with refractoriness of relapsed ALL cells to chemotherapeutic reagents.

\section{Materials and methods}

Patients and DNA samples. The ALL-BFM 2000 trial of the Berlin-Frankfurt-Münster (BFM) study group on treatment of childhood ALL enrolled patients from ages 1 to 18 years at diagnosis. Relapse patients were enrolled in the ALL-REZ BFM 2002 trial of the BFM study group on the treatment of relapsed ALL. Both trials were approved by the local Ethics Committee. Patient samples were selected according to availability of relapse samples. DNA of 14 cases at initial diagnosis, remission and relapse was extracted using standard techniques.

SNP-chip analysis. DNA from the 14 ALL samples at initial diagnosis and at relapse, as well as their paired normal DNA from remission samples were analyzed on Affymetrix ${ }^{\circledR}$ GeneChip ${ }^{\circledR}$ Human mapping 250K Nsp arrays (Affymetrix Japan, Tokyo, Japan) according to the manufacturer's protocol. Microarray data were analyzed for determination of both total and allelic-specific copy numbers using the CNAG program as previously described with minor modifications, where the status of copy numbers as well as UPD at each SNP was inferred using the algorithms based on Hidden Markov Models $(14,15)$.

Quantitative genomic PCR. Quantitative genomic PCR (qPCR) was performed on the real-time PCR machine, iCycler (Bio-Rad Laboratories, Hercules, CA) using iQ SYBR-Green supermix (Bio-Rad Laboratories) according to the manufacturer's protocol. Primer sequences used for the qPCR are; NF2-S: 5'-GAG GAG CCA ATC AGT ACA TC-3', and NF2-AS: 5'-AAC ATC ATA TCT TCC ATG AA-3'. Gene dosage at the $2 \mathrm{p}$ allele was used as an internal control as previously reported (16).

\section{Results}

Genomic abnormalities acquired at relapse. SNP-chip analysis using ALL samples and their matched normal controls allowed us to evaluate total and allele-specific (each parental) gene dosage as shown in Fig. 1a. For all patients, we examined their initial diagnosis, remission and relapse ALL samples by SNP-chip. We found a number of genomic abnormalities including deletions and duplications. Tables I and II list all of the abnormalities and representative results are shown in Fig. 1. The genomic status of ALL samples was compared at initial diagnosis, remission and relapse (Table II) to identify additional genomic abnormalities at relapse.

At initial diagnosis, two cases (\#655, \#747) showed no abnormalities over their entire genome; and both cases developed genomic abnormalities at relapse (Table II). Four of 14 cases had no additional genomic abnormality at relapse compared to initial diagnosis (Table I).
Table I. Genomic abnormalities of pediatric ALL at initial diagnosis determined by SNP-chip analysis.

\begin{tabular}{|c|c|}
\hline Case & Genomic abnormalities at diagnosis \\
\hline \#339 & $\begin{array}{l}\text { Dup 1q21.1-qter, Dup 6q14.1-pter } \\
\text { Trisomy } 4,7,8,10,14,15,17,18,21 \text {, } \\
\text { Tetrasomy X }\end{array}$ \\
\hline \#349 & $\begin{array}{l}\text { Trisomy } 4,6,14,17, \mathrm{X} \\
\text { Tetrasomy } 21\end{array}$ \\
\hline \#407 & $\begin{array}{l}\text { Del 3p25.1, Del 3q26.32, Del 6p24.1, } \\
\text { Del 7p14.1, Dup 14q12-pter, Del 14q22.1 } \\
\text { Del 18p11.31, UPD Chr. 5, UPD Chr. } 15 \\
\text { Trisomy 8, 10, 14, 16, 21 Disomy X }\end{array}$ \\
\hline \#453 & $\begin{array}{l}\text { Trisomy } 4,6,8,14,17,18, \mathrm{X}, \text { Tetrasomy } 21 \\
\text { Del 9p21.3**, Del 18q22.1 }\end{array}$ \\
\hline$\# 514^{\mathrm{a}}$ & $\begin{array}{l}\text { Del 3q26.32, Del 7q11.22-ptet, Del 7p14.1, } \\
\text { Del 9p13.2-pter**, Del 17q21.2 }\end{array}$ \\
\hline$\# 515^{\mathrm{a}}$ & Del 1q42.2, Del 7p14.1, Del 12p12.3-p13.31 \\
\hline$\# 562^{\mathrm{a}}$ & $\begin{array}{l}\text { Del 1p13.2, Trisomy } 4,6,9,10,14,18 \\
\text { Tetrasomy } 21 \text {, Disomy } X\end{array}$ \\
\hline \#581 & $\begin{array}{l}\text { Del 4q31.3, Del 6q25.1, Del 11p13, } \\
\text { Del 11p11.2, Del 11q14.2, Del 16q22.1 }\end{array}$ \\
\hline$\# 615^{\mathrm{a}}$ & $\begin{array}{l}\text { UPD Chr. 1, 2, 3, 4, 5, 6, 7, 8, 9, 10, 11, 12, } \\
\text { UPD Chr. 13, 15, 16, 17, 18, 19, 20, } 22 \\
\text { Tetrasomy 14, Tetrasomy 21, Tetrasomy X }\end{array}$ \\
\hline \#655 & Normal \\
\hline \#664 & $\begin{array}{l}\text { Del 1p12-pter, Dup 5q11.12-pter, } \\
\text { Del 6p22.1-pter, Del 6q14.3-q15, } \\
\text { Del 6q16.1-qter, Del 8q13.3-pter, } \\
\text { Dup 8q13.3-qter, Del 9q22.1-pter, } \\
\text { Del 12p12.2-pter, Del 12q21.33, } \\
\text { Del 12q24.31, Del 20q11.22 } \\
\text { Monosomy 3, 4, 7, 9, 11,12,13,15, } 20\end{array}$ \\
\hline \#672 & $\begin{array}{l}\text { Trisomy } 4,6,10,14,17,18,21, \mathrm{X} \\
\text { UPD Chr. } 9\end{array}$ \\
\hline$\# 747$ & Normal \\
\hline \#794 & $\begin{array}{l}\text { Del 6p22.3, Del 12p13.2, Del 12q21.33, } \\
\text { Dup Xq21.1-qter }\end{array}$ \\
\hline
\end{tabular}

${ }^{\mathrm{a} C a s e}, \# 514, \# 515, \# 562$, and \#615 had no additional abnormalities at relapse. Del, deletion; Dup, duplication; UPD, uniparental disomy; pter, terminal of a short arm and qter, terminal of a long arm.

Additional genomic abnormalities were noted at relapse in 10 cases (Table II). Case \#339 had no abnormality in chromosome 9p at initial diagnosis but developed a hemizygous deletion of INK4A/ARF gene on 9p at relapse (Fig. 1b and Table II). Case \#453 had a hemizygous deletion of INK4A/ARF at initial diagnosis and the matched relapsed 
a

Representative results of SNP-chip analysis

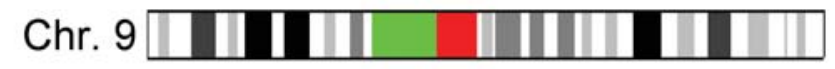
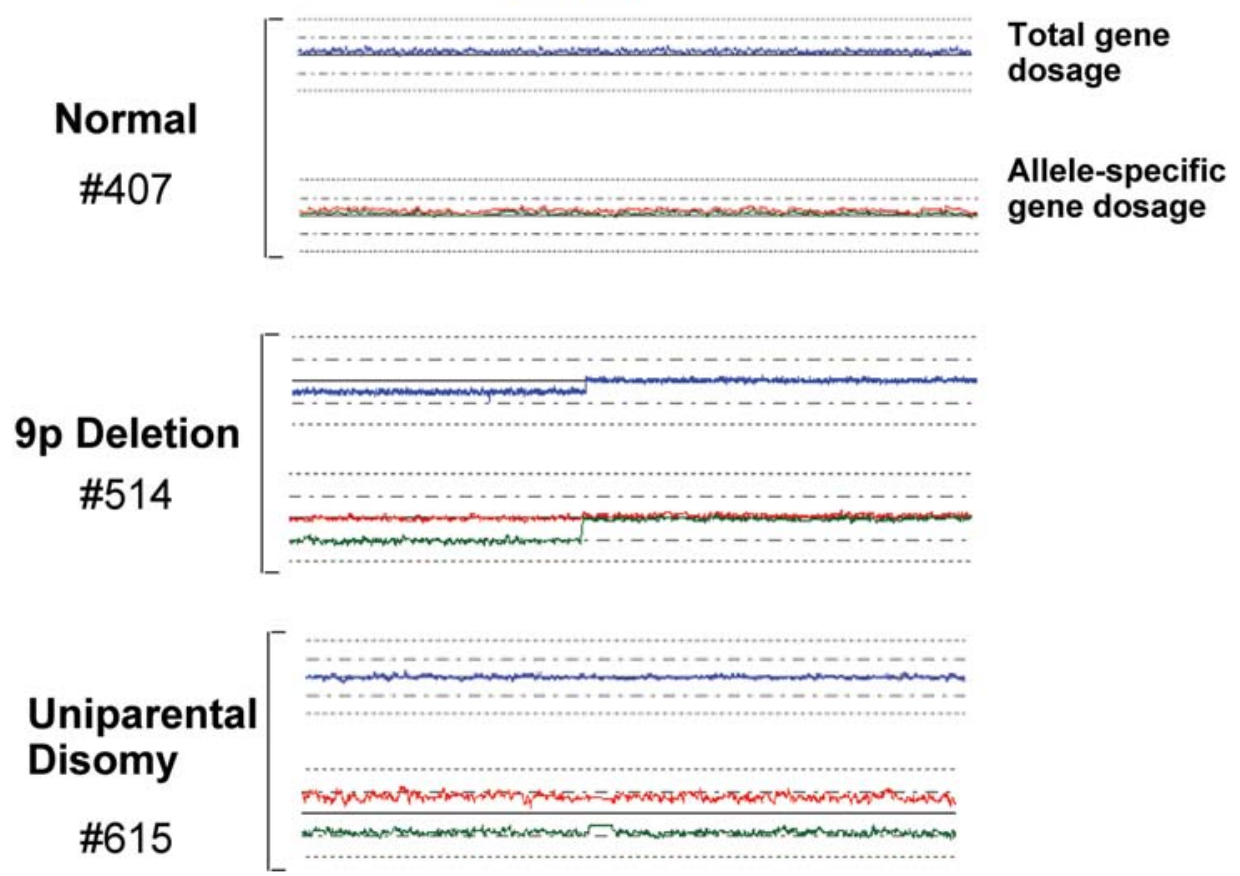

b

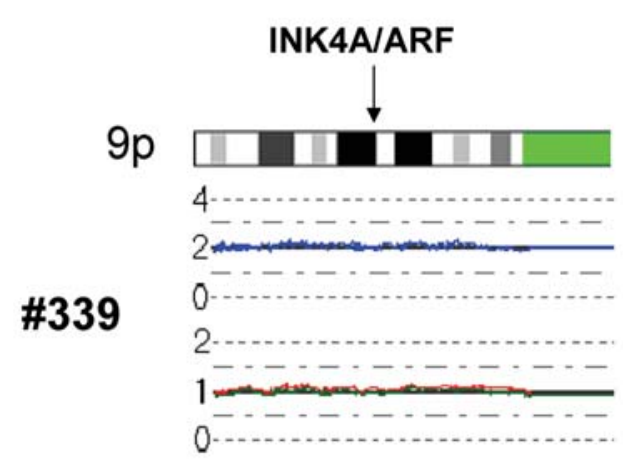

Initial Diagnosis

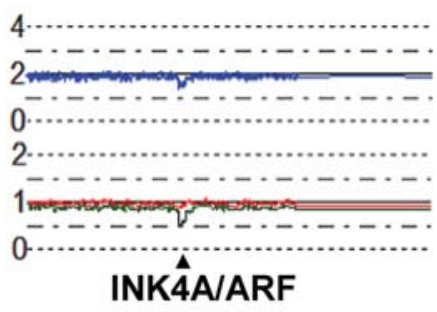

Initial Diagnosis

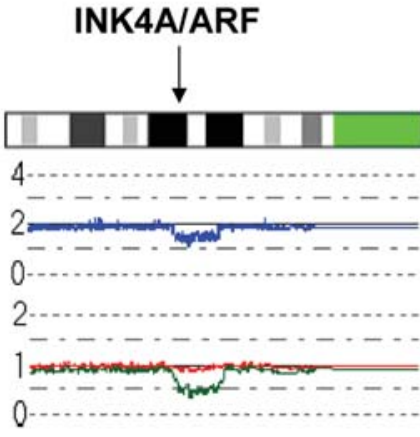

Relapse

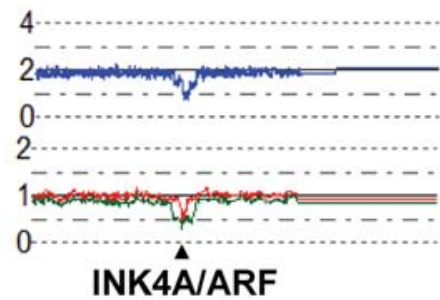

Relapse 
C

Chr. 22

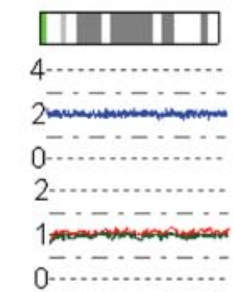

\section{Initial Diagnosis}

\#794

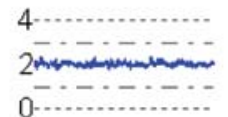

2

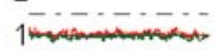

0

Initial Diagnosis

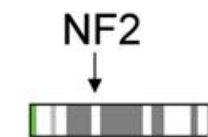

4--

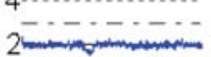

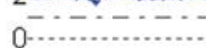

2

10

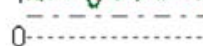

Relapse

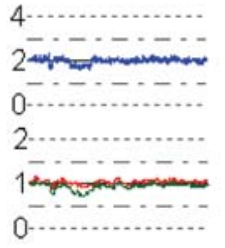

Relapse
Genomic

Real-time PCR

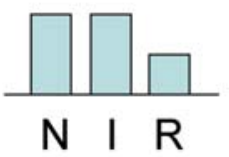

N I R

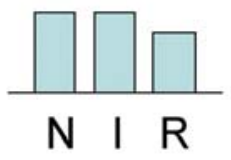

d

Chr. 16

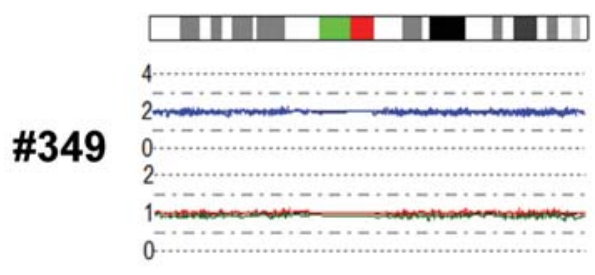

Initial Diagnosis

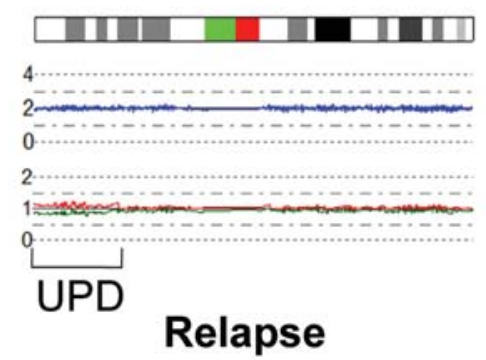

Figure 1. Representative results of SNP-chip analysis. (a) Representative results of SNP-chip analysis. Three cases (Normal: \#407, Deletion: \#514, and UPD: \#615) are shown; a blue line in each panel shows total gene dosage; a solid horizontal black line indicates diploid (2N) amount of DNA, which is normal. Green and red lines in each panel show allele-specific gene dosage levels (one indicates the gene dosage of paternal allele, the other indicates the gene dosage of maternal allele); a solid horizontal line indicates $1 \mathrm{~N}$ amount of DNA, which is normal. (a) Top panel (Normal: \#407) shows a pattern of normal chromosome 9. Blue line is at level 2 (2N DNA). Each allele-specific gene dosage (green and red lines) is at level 1N. (a) Middle panel (Deletion: \#514) shows deletion of a short arm of chromosome 9. A blue line in the short arm is lower than normal level; and one of the allele-specific gene dosage lines (green line) is lower than normal; the other allele-specific gene dosage (red line) is at normal level (1N). (a) Bottom panel (UPD: \#615) shows the pattern of uniparental disomy (UPD) detected by SNP-chip. Total gene dosage (blue line) is normal (2N). Allele-specific gene dosage data show that one allele (green line) is deleted (lower than $1 \mathrm{~N}$ ) and the other allele (red line) is duplicated (higher than $1 \mathrm{~N}$ ). Chr, chromosome. (b) INK4A/ARF deletion detected as an additional genomic change at relapse of ALL. Case \#339 had no abnormality in chr. 9p at initial diagnosis and developed hemizygous deletion of INK4A/ARF at relapse. Case \#453 had hemizygous deletion of INK4A/ARF at initial diagnosis [one of the parental alleles has deletion (green line)] and developed homozygous deletion of this gene at relapse [each parental (green or red line) allele had deletion of this region]. (c) NF2 deletion detected as an additional genomic change at relapse of ALL. Each case developed a deletion of 22q12.2 involving NF2 at relapse. Left and middle panels: Results of SNP-chip analysis at initial diagnosis (left panel) and at relapse (middle panel). Right panel: results of quantitative genomic PCR. Allelic dosages at NF2 gene were measured by real-time PCR and graphically shown. N, matched normal control DNA (remission marrow); I, ALL sample at initial diagnosis; R, ALL sample at relapse. (d) UPD detected as an additional genomic change at relapse of ALL. Case \#349 developed 16p UPD at relapse. Left panel: SNP-chip data of ALL at initial diagnosis, right panel: SNP-chip data of ALL at relapse.

sample had a homozyous deletion of this gene (Fig. 1b and Table II).

Two cases (\#581, \#794) had deletion of 22q12.2 involving NF2 as an additional genomic change at relapse (Table II and Fig. 1c). Deletions of genes associated with development of lymphocytes and other hematopoietic cells, including EBF1, ETV6, and Evi1, also appeared for the first time at relapse
(Table III). In addition, uniparental disomy (UPD) of 16p12.3-pter was detected at relapse in case \#349 (Table II and Fig. 1d).

Disappearance of deletions at relapse. Of interest, loss of genomic materials (deletions and monosomy) detected at initial diagnosis disappeared at relapse in four cases $(\# 407$, 
Table II. New genomic changes in matched relapsed ALL samples.

\begin{tabular}{|c|c|c|}
\hline \multicolumn{3}{|c|}{ At Relapse } \\
\hline & New abnormality & Disappearance of abnormality \\
\hline \#339 & $\begin{array}{l}\text { UPD chr. } 15 \\
\text { Del 9p21.3 }\end{array}$ & Trisomy $7,14,17$ \\
\hline \#349 & $\begin{array}{l}\text { UPD chr. X } \\
\text { UPD 16p12.3-pter }\end{array}$ & Trisomy 4, 17 \\
\hline \#407 & $\begin{array}{l}\text { Del 10q11.23, Del 10q23.33-q24. } 2 \\
\text { Del 11q21, Del 16q23.1, } \\
\text { Del 19q13.31-q13.33, } \\
\text { Del 21q21.1-q21.2 }\end{array}$ & Del 14q22.1 \\
\hline \#453 & $\begin{array}{l}\text { Trisomy } 16 \\
\text { Del 9p21.3 }\end{array}$ & Del 18q22.1 \\
\hline \#581 & $\begin{array}{l}\text { Del 12p12.2-p13.2, Del 12p13, } \\
\text { Del 12p11.1, Del 12q21.31-q21.32, } \\
\text { Del 18q12.2-q12.3, Del 18q12.3, } \\
\text { Del 18q21.32, Del 18q23, Del 22q12. } 2\end{array}$ & Del 4q31.3 \\
\hline \#655 & Del 1q42.12-q44, Del 10q26.3 & \\
\hline \#664 & $\begin{array}{l}\text { Del 2p12-p15, Del 5q33.3 } \\
\text { Del 12p13.2, Del 12p13.1-p13.3 } \\
\text { Del 20q11.22 }\end{array}$ & $\begin{array}{l}\text { Del 1p12-pter } \\
\text { Monosomy 3, 4, } 20\end{array}$ \\
\hline \#672 & Dup 9p24.1-pter & \\
\hline \#747 & Del 7p11.2-pter, Dup 7q11.21-qter & \\
\hline \#794 & $\begin{array}{l}\text { Del 1p36.11, Del 2p31.21, Del 3q26.2, } \\
\text { Dup 8q12.1-qter, Dup 12p13.1-pter, } \\
\text { Dup 21q22.12-pter, Del22q12.1-q12.2 }\end{array}$ & \\
\hline
\end{tabular}

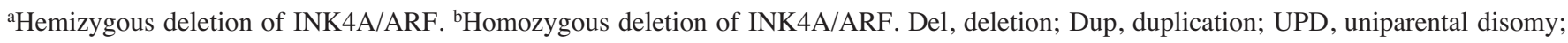
pter, terminal of a short arm and qter, terminal of a long arm.

Table III. Candidate genes present in deletions found only in matched relapsed samples.

\begin{tabular}{llr}
\hline Candidate gene & Abnormality (case no.) & Nucleotide position \\
\hline INK4A/ARF & Del 9p21.3(\#339) & $19,949,158-25,971,645$ \\
& Del 9p21.3(\#453) & $21,270,454-23,049,968$ \\
NF2 & Del 22q12.2 (\#581) & $27,847,323-30,706,527$ \\
& Del 22q12.1-q12.2(\#794) & $25,335,051-28,723,971$ \\
EBF1 & Del 5q33.3 (\#664) & $158,185,016-158,492,732$ \\
ETV6 & Del 12p13.2 (\#664) & $11,553,486-12,209,757$ \\
Evi1 & Del 3q26.2 (\#794) & $170,227,876-171,017,968$ \\
\hline
\end{tabular}

\#453, \#581, \#664) (Table II). For example, while case \#581 had deletion of $4 \mathrm{q} 31.3$ at initial diagnosis, this abnormality disappeared at relapse (Table II and Fig. 2a). Nevertheless, other chromosomal abnormalities including del $6 \mathrm{q}$, del 11p, del 11q and del 16q were present both at diagnosis and relapse (Table I, Fig. 2a and data not shown). Case \#664 had a deletion at $1 \mathrm{p}$ at initial diagnosis, but this region appeared normal at relapse (Table II and Fig. 2b) while other genomic 
a

\#581

$4 q$

Del $4 q 31.3$

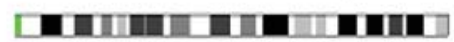

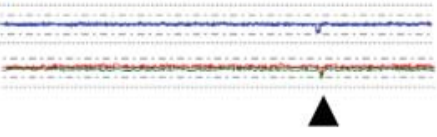

Initial Diagnosis

Del 6q25.1

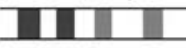

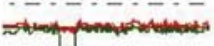

Initial Diagnosis

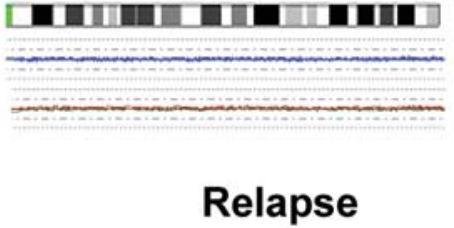

Del 6q25.1

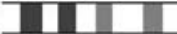

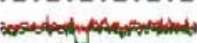

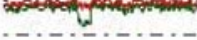

Relapse

b

\#664

$1 p$

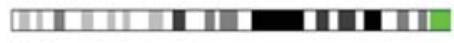

2

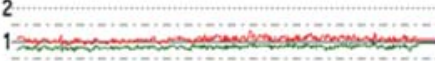

Deletion

Initial Diagnosis

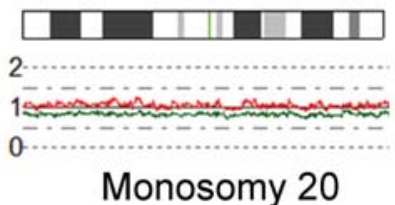

Initial Diagnosis
Relapse
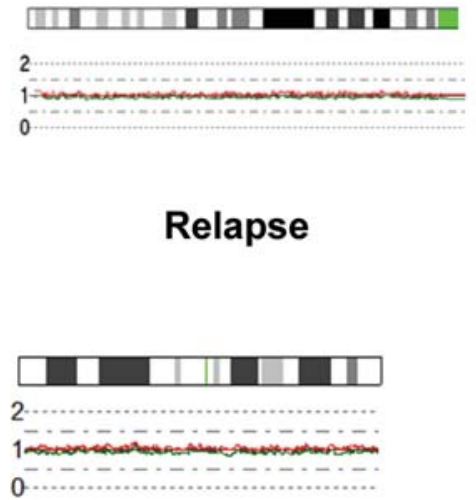

Chr. 20 normal

Relapse
Del 6q16.1-qter

Initial Diagnosis
Del 6q16.1-qter

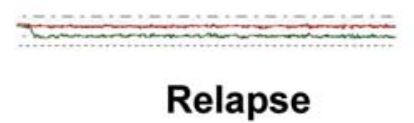

Figure 2. Genomic abnormalities at diagnosis of ALL is absent at relapse. (a) Case \#581 had deletion of 4q31.3 at initial diagnosis (arrow head), which disappeared at relapse (upper panel). The deletion of 6q25.1 was detected at both initial diagnosis and relapse in this case (lower panel). (b) Case \#664 had a deletion in 1p region at diagnosis; in contrast, no abnormalities in this region were found at relapse (top panel). This case also had monosomy 20 at initial diagnosis, and normal disomy of chromosome 20 at relapse (middle panel). Deletion of 6q16.1-qter was detected at both initial diagnosis and relapse (bottom panel). 


\section{\#339}
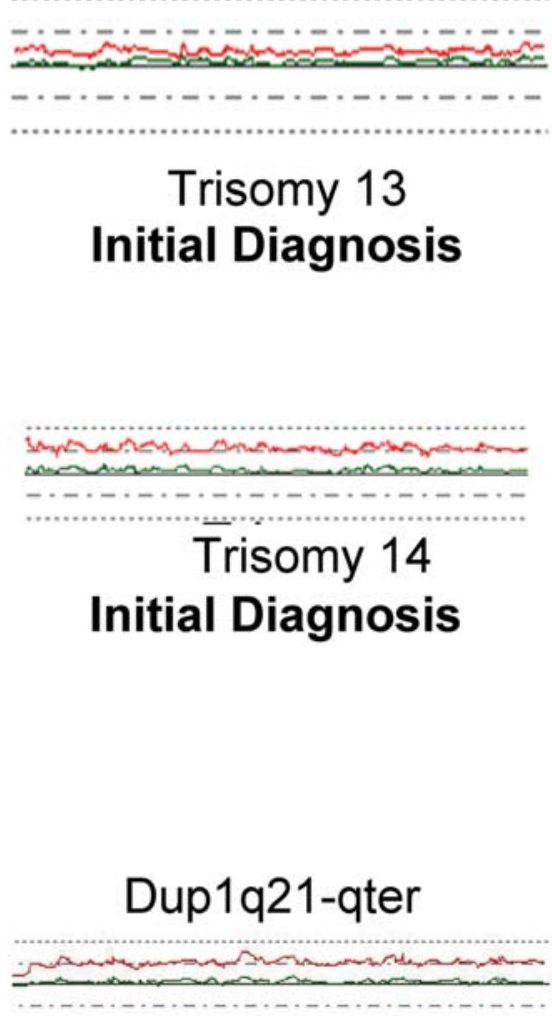

Initial Diagnosis
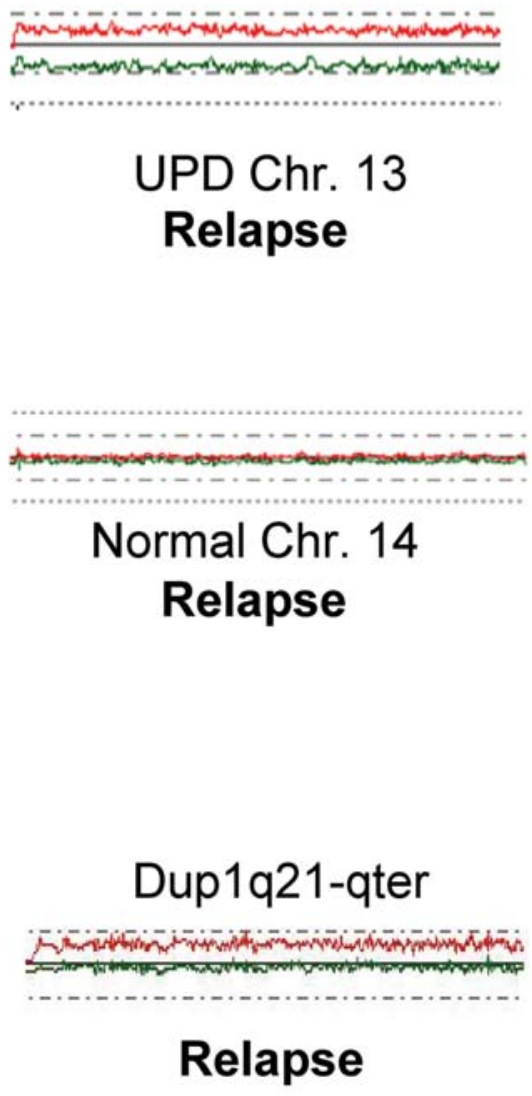

Figure 3. Trisomy at initial diagnosis changed to either uniparental or normal disomy at relapse. ALL sample of case \#339 had trisomy 13 at initial diagnosis; same region changed to UPD at chromosome 13 at relapse (top panel); same sample of ALL at initial diagnosis had trisomy 14; but at relapse, chromosome 14 became normal disomy (middle panel). Duplication of 1q21-qter was detected at both initial diagnosis and relapse in this case (bottom panel).

abnormalities including del 6q16.1-qter were detected both at initial diagnosis and relapse (Table I and Fig. 2b). Furthermore, case \#664 also had monosomies of chromosomes 3, 4, and 20 at initial diagnosis, but had a normal appearing genome in these chromosomes at relapse (Table II, Fig. 2b).

Numerical chromosomal abnormalities and whole chromosome UPD. At initial diagnosis, gain of whole chromosomes including trisomy and tetrasomy, were detected in 7 cases (\#339, \#349, \#407, \#453, \#562, \#615, \#672) and loss of whole chromosomes occurred in one case (\#664) (Table I). In three cases (\#339, \#349, \#664), these numerical changes disappeared at relapse in several chromosomes (Table II). In two of these cases (\#339, \#349), trisomy changed to whole chromsome UPD [trisomy X to UPD chr. X (\#349) and trisomy 15 changed to UPD chr. 15 (case \#339)] (Table II, Fig. 3 top panel and data not shown). In two cases (\#339,\#349), trisomy reverted to normal genomic appearance of several chromosomes (Table II, Fig. 3 middle panel and data not shown). In these cases, other genomic abnormalities including dup 1q21-qter (\#339) were detected at both initial diagnosis and relapse. One unusual case (case \#615) had UPD for each of 20 chromosomes at both initial diagnosis and relapse and had no additional genomic change at relapse (Table I).
Disruption of PTPRD gene. Case \#672 had whole chromosome 9 UPD at initial diagnosis (Table I and Fig. 4a) and this UPD was lost at relapse. Instead of UPD, this case had duplication of 9p13-pter at relapse (Fig. 4a). SNP-chip data defined the start-site of this duplication as intron 23 of PTPRD gene (Fig. 4b and c), suggesting that this gene was disrupted in the ALL cells at relapse.

\section{Discussion}

Most children with ALL are cured in large part because of progressively better drugs and their mode of administration (2-4). Nevertheless, $\sim 15-20 \%$ of the children relapse and their overall disease-free survival is poor $(10,11)$. An unmet need is either to recognize these children at diagnosis and provide them alternative therapy and/or recognize new genomic therapeutic targets in the relapsed ALL cells that might hold the key for better relapse-related therapies. We previously examined the genomic abnormalities of ALL cells observed at diagnosis of 399 children and attempted to prognosticate who would relapse (16). Except for children who had hyperdiploid ALL with normal chromosomes 17/18 or either BCR-ABL positive ALL or NUP214-ABL positive ALL, no other obvious genomic changes at diagnosis foretold who would relapse (16). 


\section{9p (\#672)}

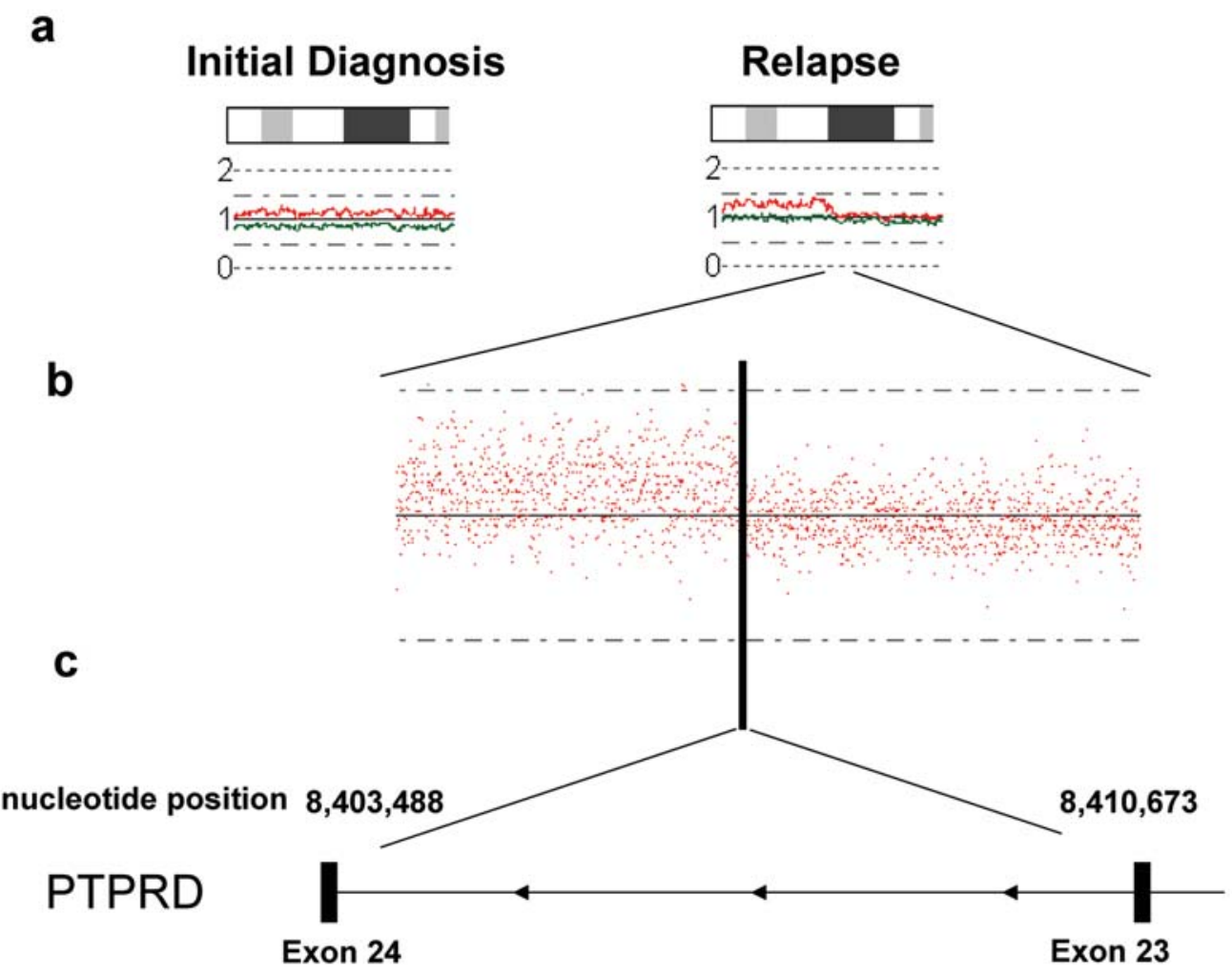

Figure 4. Disruption of PTPRD gene in relapsed ALL. (a) Duplication of 9p detected as an additional genomic abnormality at relapse of ALL. At initial diagnosis, case \#672 had UPD at 9p (left panel) which changed to duplication of 9p24.1-pter at relapse (right panel). (b) Magnified view of SNP-chip data. Relapsed sample: signals of individual SNP probes around 9p24.1 are represented by dots. A vertical line indicates the start-site of duplication of 9p24.1-pter. (c) PTPRD gene involved at the start-site of duplication 9p24.1-pter. The duplication starts at intron 23 of PTPRD gene. Black boxes indicate exons of PTPRD gene. Arrows indicate the direction of transcription of the PTPRD gene. Nucleotide positions of SNP-probes closest to the breakpoint are shown.

In this study, molecular allelokaryotyping was performed on 14 relapsed pediatric ALL. We validated the SNP-chip results using real-time quantitative PCR at a number of deleted sites identified by SNP-chip (Fig. 1c and data not shown). The real-time quantitative PCR data were congruent with the findings of SNP-chip at all sites that we examined. Furthermore, in other leukemia samples we validated the techniques using FISH (17). These data suggested that SNP-chip analysis was reliable.

Genomic status of these ALL samples was compared with ones at initial diagnosis. Additional genetic changes were discovered at relapse in ten of 14 relapsed ALL cases. We found that acquired genomic abnormalities were common events at relapse of pediatric ALL.

Interestingly, in four cases, loss of genomic materials (deletions/monosomy) detected at initial diagnosis, disappeared at relapse. This suggests that the dominant ALL clones at diagnosis had these deletions; and other clones which did not have these changes were minor undetectable clones at diagnosis and emerged at relapsed. These minor clones may have been chemotherapy-resistant even at initial diagnosis. If the resistant clones are not detectable at initial diagnosis, predicting prognosis based on the genomic changes identified in the dominant clones will not be very successful. Defining genetic features of resistant clones and having the ability sensitively to detect these minor resistant clones at initial diagnosis, will enhance our ability to predict prognosis of patients and select therapy for these more resistant clones.

In two cases (\#339, \#349), trisomy 17 at initial diagnosis changed to normal appearing chromosome 17 at relapse. As mentioned above, we have found that hyperdiploid ALL with normal status of chromosome 17 have a worse prognosis than those with hyperdiploid and trisomy $17(16,18,19)$. Taken together, refractoriness to chemotherapeutic reagents by these cells might reflect changing trisomy 17 to normal chromosome 17.

In two cases (\#339, \#349), trisomy at initial diagnosis $(+15,+\mathrm{X}$, respectively) changed to UPD at relapse; and as described above, trisomy at initial diagnosis also changed to normal disomy in several chromosomes at relapse. One of the three chromosomes was lost at relapse in these cases and changed to uniparental disomy or normal disomy. This may suggest that one of three chromosomes was randomly lost at relapse. UPD may not be associated with a growth advantage for cancer cells, but merely represents a random event caused by chromosomal instability. Alternatively, a duplication of either a mutation or a polymorphic site with the loss of the other normal allele may provide a drug-resistant phenotype $(15,20)$.

Deletions of INK4A/ARF and NF2 were detected as additional genomic changes in two cases. Although the observation is intriguing, the number of cases that we have 
examined is too small to show any statistical significance. Our previous SNP-chip analysis of 399 pediatric ALL identified neither deletion of INK4A/ARF nor deletion of NF2 as an adverse factor for the prognosis (16). We found a number of other candidate genes involved in additional genomic abnormalities in individual cases at relapse, including deletion of EBF1, ETV6, and Evi1 (Table III). Deletions of these genes were also detected in ALL samples at initial diagnosis. To our knowledge, these abnormalities have not been associated with refractory ALL.

The commonly deleted region on 9p involves INK4A/ ARF in ALL (16,21); PAX5 gene is also involved in this deletion in a number of ALL cases $(16,22)$. Deletion of $9 p$ in ALL is sometimes larger and involves PTPRD. We found disruption of the PTPRD gene at relapse, which was not detected at initial diagnosis. This may suggest that the PTPRD gene is translocated to another gene and generate a novel fusion gene (23).

Deletion and/or rearrangement of PTPRD have been reported in other cancers including melanoma (24), neuroblastoma (25), cutaneous squamous cell carcinoma (26) and lung cancers (27-29). PTPRD is localized on 9p, and this region is frequently deleted in ALL (11). PTPRD is a receptortype tyrosine phosphatase which plays an important role in neuronal tissues $(30,31)$. The gene is highly expressed in normal B-cells $(30,31)$; dysregulation of PTPRD may be associated with leukemogenesis. Mutations found in cancer cells have been classified into two types, 'driver' and 'passenger' mutations; 'driver' mutations are associated with carcinogenesis and 'passenger' mutations 'come along for the ride' but are not oncogenic (32). Further studies are needed to clarify the importance of disruption of PTPRD and its association with leukemogenesis.

During the preparation of this study, SNP-chip analysis of relapse ALL by other investigators has been published (33). They also found several acquired genomic abnormalities at relapse (33).

This study defined a number of new findings which will help further analysis of genetic abnormalities in relapsed ALL. Analysis of the additional data of acquired genomic abnormalities of relapsed pediatric ALL will allow us to recognize a genomic signature strongly associated with poor prognosis which will modify our therapy and also help us to identify new therapeutic targets.

\section{Acknowledgements}

We thank the Parker Hughes Fund and NIH grants for supporting this study. NK is supported by the fellowship from The Tower Cancer Research Foundation. HPK holds the Mark Goodson Chair in Oncology Research at Cedars Sinai and is a member of the Jonsson Cancer Center and the Molecular Biology Institute of UCLA. This study was also supported by grant-in-aid from Department of Health, Welfare and Labor and from MEXT of the Japanese government, by EU grant FOOD-CT-2005-016320 and a grant by the Deutsche Krebshilfe to CRB. The ALL-BFM 2000 trial is supported by $50-2698-S c h r 1$ of the Deutsche Krebshilfe. The ALL-REZ BFM 2002 trial is supported by the Deutsche Kinderkrebsstiftng. The study is dedicated to David Golde, a mentor and friend.

\section{References}

1. Pui $\mathrm{CH}$, Relling MV and Downing JR: Acute lymphoblastic leukemia. N Engl J Med 350: 1535-1548, 2004.

2. Pui $\mathrm{CH}$ and Evans WE: Acute lymphoblastic leukemia. N Engl J Med 339: 605-615, 1998.

3. Creutzig U, Ritter J, Zimmermann M, et al: Improved treatment results in high-risk pediatric acute myeloid leukemia patients after intensification with high-dose cytarabine and mitoxantrone: results of Study Acute Myeloid Leukemia-Berlin-FrankfurtMunster 93. J Clin Oncol 19: 2705-2713, 2001.

4. Schrappe M, Reiter A, Ludwig WD, et al: Improved outcome in childhood acute lymphoblastic leukemia despite reduced use of anthracyclines and cranial radiotherapy: results of trial ALL-BFM 90. German-Austrian-Swiss ALL-BFM Study Group. Blood 95: 3310-3322, 2000

5. Hongo T, Yajima S, Sakurai M, Horikoshi Y and Hanada R: In vitro drug sensitivity testing can predict induction failure and early relapse of childhood acute lymphoblastic leukemia. Blood 89: 2959-2965, 1997

6. Kaspers GJ, Veerman AJ, Pieters R, et al: In vitro cellular drug resistance and prognosis in newly diagnosed childhood acute lymphoblastic leukemia. Blood 90: 2723-2729, 1997.

7. Holleman A, Cheok MH, den Boer ML, et al: Gene-expression patterns in drug-resistant acute lymphoblastic leukemia cells and response to treatment. N Engl J Med 351: 533-542, 2004.

8. Lugthart S, Cheok MH, den Boer ML, et al: Identification of genes associated with chemotherapy crossresistance and treatment response in childhood acute lymphoblastic leukemia. Cancer Cell 7: 375-386, 2005

9. Armstrong SA, Staunton JE, Silverman LB, et al: MLL translocations specify a distinct gene expression profile that distinguishes a unique leukemia. Nat Genet 30: 41-47, 2002.

10. Borgmann A, von Stackelberg A, Hartmann R, et al: Unrelated donor stem cell transplantation compared with chemotherapy for children with acute lymphoblastic leukemia in a second remission: a matched-pair analysis. Blood 101: 3835-3839, 2003.

11. Einsiedel HG, von Stackelberg A, Hartmann R, et al: Long-term outcome in children with relapsed ALL by risk-stratified salvage therapy: results of trial acute lymphoblastic leukemia-relapse study of the Berlin-Frankfurt-Munster Group 87. J Clin Oncol 23: 7942-7950, 2005.

12. Pinkel D and Albertson DG: Array comparative genomic hybridization and its applications in cancer. Nat Genet 37: S11-S7, 2005.

13. Gunderson KL, Steemers FJ, Lee G, Mendoza LG and Chee MS: A genome-wide scalable SNP genotyping assay using microarray technology. Nat Genet 37: 549-554, 2005.

14. Nannya Y, Sanada M, Nakazaki K, et al: A robust algorithm for copy number detection using high-density oligonucleotide single nucleotide polymorphism genotyping arrays. Cancer Res 65: 6071-6079, 2005

15. Yamamoto G, Nannya Y, Kato M, et al: Highly sensitive method for genomewide detection of allelic composition in nonpaired, primary tumor specimens by use of affymetrix single-nucleotidepolymorphism genotyping microarrays. Am J Hum Genet 81: 114-126, 2007

16. Kawamata N, Ogawa S, Zimmermann M, et al: Molecular allelokaryotyping of pediatric acute lymphoblastic leukemias by high resolution single nucleotide polymorphism oligonucleotide genomic microarray. Blood 111: 776-784, 2008.

17. Lehmann S, Ogawa S, Raynaud SD, et al: Molecular allelokaryotyping of early stage untreated chronic lymphocytic leukemia. Cancer 112: 1296-1305, 2008.

18. Sutcliffe MJ, Shuster JJ, Sather HN, et al: High concordance from independent studies by the Children's Cancer Group (CCG) and Pediatric Oncology Group (POG) associating favorable prognosis with combined trisomies 4, 10, and 17 in children with NCI Standard-Risk B-precursor Acute Lymphoblastic Leukemia: a Children's Oncology Group (COG) initiative. Leukemia 19: 734-740, 2005.

19. Heerema NA, Sather HN, Sensel MG, et al: Prognostic impact of trisomies of chromosomes 10,17 , and 5 among children with acute lymphoblastic leukemia and high hyperdiploidy $(>50$ chromosomes). J Clin Oncol 18: 1876-1887, 2000.

20. Fitzgibbon J, Smith LL, Raghavan M, et al: Association between acquired uniparental disomy and homozygous gene mutation in acute myeloid leukemias. Cancer Res 65: 9152-9154, 2005.

21. Hirama T and Koeffler HP: Role of the cyclin-dependent kinase inhibitors in the development of cancer. Blood 86: 841-854, 1995. 
22. Mullighan CG, Goorha S, Radtke I, et al: Genome-wide analysis of genetic alterations in acute lymphoblastic leukaemia. Nature 446: 758-764, 2007.

23. Kawamata N, Ogawa S, Zimmermann M, et al: Cloning of genes involved in chromosomal translocations by high-resolution single nucleotide polymorphism genomic microarray. Proc Natl Acad Sci USA 105: 11921-11926, 2008.

24. Stark M and Hayward N: Genome-wide loss of heterozygosity and copy number analysis in melanoma using high-density single-nucleotide polymorphism arrays. Cancer Res 67: 2632-2642, 2007.

25. Stallings RL, Nair P, Maris JM, et al: High-resolution analysis of chromosomal breakpoints and genomic instability identifies PTPRD as a candidate tumor suppressor gene in neuroblastoma. Cancer Res 66: 3673-3680, 2006

26. Purdie KJ, Lambert SR, Teh MT, et al: Allelic imbalances and microdeletions affecting the PTPRD gene in cutaneous squamous cell carcinomas detected using single nucleotide polymorphism microarray analysis. Genes Chromosomes Cancer 46: 661-669, 2007.

27. Sato M, Takahashi K, Nagayama K, et al: Identification of chromosome arm $9 \mathrm{p}$ as the most frequent target of homozygous deletions in lung cancer. Genes Chromosomes Cancer 44 : 405-414, 2005.
28. Nagayama K, Kohno T, Sato M, Arai Y, Minna JD and Yokota J: Homozygous deletion scanning of the lung cancer genome at a 100-kb resolution. Genes Chromosomes Cancer 46: 1000-1010, 2007.

29. Zhao X, Weir BA, LaFramboise T, et al: Homozygous deletions and chromosome amplifications in human lung carcinomas revealed by single nucleotide polymorphism array analysis. Cancer Res 65: 5561-5570, 2005.

30. Uetani N, Kato K, Ogura H, et al: Impaired learning with enhanced hippocampal long-term potentiation in PTPdeltadeficient mice. EMBO J 19: 2775-2785, 2000.

31. Pulido R, Serra-Pages C, Tang M and Streuli M: The LAR/PTP delta/PTP sigma subfamily of transmembrane protein-tyrosinephosphatases: multiple human LAR, PTP delta, and PTP sigma isoforms are expressed in a tissue-specific manner and associate with the LAR-interacting protein LIP.1. Proc Natl Acad Sci USA 92: 11686-11690, 1995.

32. Haber DA and Settleman J: Cancer: drivers and passengers. Nature 446: 145-146, 2007.

33. Mullighan CG, Phillips LA, Su X, Ma J, Miller CB, Shurtleff SA and Downing JR: Genomic analysis of the clonal origins of relapsed acute lymphoblastic leukemia. Science 322: 1377-1380, 2008. 